\title{
Erratum to: Ultrasound exposure (mechanical index 1.8) with acoustic radiation force impulse evokes extrasystolic waves in rabbit heart under concomitant administration of an ultrasound contrast agent
}

\author{
Yasunao Ishiguro $^{1}$ - Naotaka Nitta ${ }^{2}$ Nobuyuki Taniguchi ${ }^{3} \cdot$ Kazuki Akai $^{4}$. \\ Noriya Takayama $^{3} \cdot$ Hideki Sasanuma $^{1}$ Yukiyo Ogata ${ }^{5} \cdot$ Yoshikazu Yasuda $^{1}$. \\ Iwaki Akiyama $^{4}$
}

Published online: 28 October 2015

(C) The Japan Society of Ultrasonics in Medicine 2015

\section{Erratum to: J Med Ultrasonics \\ DOI 10.1007/s10396-015-0654-0}

The fifth author's name appeared incorrectly in the original article. The correct author's name is given here.

The online version of the original article can be found under doi:10.1007/s10396-015-0654-0.

Yasunao Ishiguro

ynao-i@ mub.biglobe.ne.jp

1 Department of Surgery, Jichi Medical University, School of Medicine, 3311-1 Yakushiji, Shimotsuke, Tochigi 329-0498, Japan

2 Human Technology Research Institute, National Institute of Advanced Industrial Science and Technology (AIST), Tsukuba, Ibaraki, Japan

3 Department of Clinical Laboratory Medicine, Jichi Medical University, School of Medicine, Shimotsuke, Tochigi, Japan

4 Medical Ultrasound Research Center, Doshisha University, Kyotanabe, Kyoto, Japan

5 Division of Cardiovascular Medicine, Department of Medicine, Jichi Medical University, School of Medicine, Shimotsuke, Tochigi, Japan 